\title{
The Analysis of Braess' Paradox and Robustness Based on Dynamic Traffic Assignment Models
}

\author{
Bai-Bai Fu, ${ }^{1}$ Chun-Xue Zhao, ${ }^{2}$ and Shu-bin $\mathrm{Li}^{3}$ \\ ${ }^{1}$ School of Architecture and Urban Planning, Shandong Jianzhu University, Jinan 250101, China \\ ${ }^{2}$ School of Mathematics and Statistics, Anyang Normal University, Anyang 455000, China \\ ${ }^{3}$ Public Security Department, Shandong Police College, Jinan 250014, China \\ Correspondence should be addressed to Bai-Bai Fu; fubaibai@163.com
}

Received 25 July 2013; Accepted 24 October 2013

Academic Editor: Wuhong Wang

Copyright (C) 2013 Bai-Bai Fu et al. This is an open access article distributed under the Creative Commons Attribution License, which permits unrestricted use, distribution, and reproduction in any medium, provided the original work is properly cited.

\begin{abstract}
The investigation of the paradox and robustness about the traffic network is an important branch of the traffic assignment. In this paper, Braess' paradox and robustness of the dynamic traffic network are analyzed by the dynamic traffic assignment models. In addition, the relationship of total costs with different traffic assignment models is discussed. The results show that the paradox only occurs in certain range; the robustness of the network and the relationship of total traffic costs are changed as the traffic demand changes, which provides theoretical guidance for the urban transportation planning.
\end{abstract}

\section{Introduction}

In recent years, the proposal of the intelligent transportation system (ITS) forces people to investigate the dynamic traffic assignment (DTA). The mathematical programming $[1,2]$, the optimal control $[3,4]$, and the variational inequality (VI) $[5,6]$ were proposed, respectively, and labeled as three main analytical approaches. Specially, the optimal control includes dynamic user equilibrium (DUE) [7-11] and dynamic system optimal (DSO) [12].

Braess' paradox is an important phenomenon during the traffic assignment [13], which has been widely investigated in the scientific literatures [14-16], such as X. Zhang and H. M. Zhang [17] studied the route choices and a novel paradox in queuing networks; Pas and Principio [18] gained the specific range that the paradox occurs when the link cost only depends on the link flow. Braess' paradox is rooted in the essence of the user equilibrium (UE) assignment where each user minimizes his/her own travel time between an origin and a destination. The most previous work mainly focuses on the paradox under the static traffic. In recent years, the paradox of the dynamic traffic assignment began to develop, such that Arnott et al. [19] discussed the properties of the dynamic traffic equilibrium including a paradox; Hallefjord et al. [20] analyzed the traffic paradox when the travel demand is elastic; Zhang et al. [21] investigated Braess' paradox in the dynamic traffic assignment. In this paper, we investigate the paradox phenomenon of the dynamic traffic network using VI method and assuming the link cost at time $t$ is related to the flow of this link and the adjacent links at the same paths at time $t$.

The robustness, as an important index valuing the stability of the system, is usually used to study the network under the partial degradation. In the process of studying the robustness, it is generally classified into the following two classes according to whether the system structure remains intact: (i) certain component of the network is removed; (ii) no component is removed, but the function of certain portion of the network falls into the perturbations. Based on different classes, different network function measures are proposed to examine the robustness of the network. In this paper, we use the relative total cost index to measure the robustness of the dynamic network considering the influence of the flow on other links when certain component (node or link) is removed.

The paper is organized as follows. We first briefly recall main analytical models of the traffic assignment; then we discuss whether the paradox occurs or not under the given link cost and investigate the relationship between the total 
cost under DUE and that under DSO; subsequently, we investigate the robustness of the dynamic network; at last, the conclusions about the results of the paper are given.

\section{Main Analytical Models}

2.1. The DUE. In this section, we briefly reviewed dynamic equilibrium models [8-11] of the traffic network. Assume that the traffic network includes a set $W$ of origin/destination (O/D) pairs with $n_{W}$ elements and a set $P$ of paths joining $\mathrm{O} / \mathrm{D}$ pairs; DUE is based on the behavioral assumption that only the minimum cost routes are used at each time $t$, whose mathematical expression is given as follows:

$$
\begin{aligned}
& C_{p}\left(x^{*}(t)\right)=\lambda_{w}(t), \quad \text { if } x_{p}^{*}(t)>0, \\
& C_{p}\left(x^{*}(t)\right) \geq \lambda_{w}(t), \quad \text { if } x_{p}^{*}(t)=0,
\end{aligned}
$$

where $C_{p}(\cdot)$ is the cost on the path $p$ under the DUO, $\lambda_{w}(t)$ is the minimal path costs at time $t, x^{*}(t)$ is a path flow pattern satisfying the flow conservation at time $t$, and $[0, T]$ denotes the time interval under consideration.

Under the equilibrium state, the link flows and the route flows satisfy the following conservation of flow equations:

$$
f_{a}(t)=\sum_{p \in P} x_{p}(t) \delta_{a p}, \quad \forall a \in L,
$$

where $f_{a}(t)$ is the flow on the link $a, x_{p}(t)$ is the flow on the rout $p, \delta_{a p}=1$ if link $a$ is contained in route $p$, and $\delta_{a p}=0$, otherwise. The traffic demand at time $t$ must satisfy the following conservation of flow:

$$
d_{w}(t)=\sum_{p \in P_{w}} x_{p}(t), \quad \forall w \in W .
$$

In addition, the model meets the following nonnegative constraint and boundary initial condition:

$$
x_{a}(t) \geq 0, \quad x_{a}(0)=0 .
$$

2.2. The DSO. DSO calls for the flow pattern that minimizes the total travel time in the system at each time $t$, whose mathematical expression is shown as follows:

$$
\begin{aligned}
& C_{p}^{\prime}\left(x^{*}(t)\right)=\mu_{w}(t), \quad \text { if } x_{p}^{*}(t)>0, \\
& C_{p}^{\prime}\left(x^{*}(t)\right) \geq \mu_{w}(t), \quad \text { if } x_{p}^{*}(t)=0,
\end{aligned}
$$

where $C_{p}^{\prime}(\cdot)$ is the cost on the path $p$ under the DSO and $\mu_{w}(t)$ is the minimal marginal path costs at time $t$. The constraint under the DSO is the same as that under DUE and is omitted here.

In addition, the link costs under DUE and DSO satisfy the relationship as follows:

$$
c_{a}^{\prime}\left(f_{a}(t)\right)=c_{a}\left(f_{a}(t)\right)+f_{a}(t) \frac{d c_{a}\left(f_{a}(t)\right)}{d f_{a}(t)},
$$

where $c_{a}(\cdot), c_{a}^{\prime}(\cdot)$ are costs on link $a$ under DUE and DSO, respectively; the link cost under the DSO is also known as the marginal link cost.
2.3. The Variational Inequalities Equivalent to DUE. Compared with the mathematical programming and optimal control, the variational inequality is the more effective way to describe the dynamic traffic assignment problem. The extensive investigation has gone ahead, such that Frieza and Smith proposed the variational inequality of the dynamic assignment program based on the path cost; Ran and Boyce proposed the variational inequality of the dynamic user optimal model based on the link cost. DUO based on the path cost is equivalent to the variational inequality as follows:

$$
\int_{0}^{T} \sum_{r s} \sum_{p} \lambda_{p}^{r s *}(t)\left[f_{p}^{r s}(t)-f_{p}^{r s *}(t)\right] d t \geq 0
$$

where $\lambda_{p}^{r s *}(t)$ is the path cost on $\mathrm{O} / \mathrm{D}$ pair $r s$ at time $t$ satisfying DUO, $f_{p}^{r s}(t)$ is the path flow on O/D pair $r s$ at time $t$, and $f_{p}^{r s *}(t)$ is the path flow on O/D pair $r s$ at time $t$ satisfying DUE.

\section{Application of Analytical Models}

\subsection{The Analysis about Paradox}

3.1.1. The Equilibrium Solution of the Four-Link Network under DUE. As we know, the paradox phenomenon exists in many networks with different topologies; Braess' network has been investigated by a large number of researchers; it is representative because it can simplify the problem and clearly explain traffic phenomena. In this paper, we also consider Braess' network with a single origin $o$ and a single destination $r$ in this paper. Figure 1 depicts the network before the addition of the new link $p q$. Let the total demand for the travel from origin $o$ to destination $r$ be $d_{w}(t)$ and $d_{w}(t)=t$; further, assume that the link cost is a function of the flow not only on this link but also on other links. Because the influence of the adjacent links is dominant, we assume that the other links are only limited to the adjacent links on the same path in this work. At the same time, assume that the problem is symmetric; further, the travel time is link-flow dependent and it increases rapidly as a function of the flow on the link.

Specifically, the link cost functions of the four-link network in Figure 1 are

$$
\begin{gathered}
c_{o p}(t)=10\left(\gamma f_{o p}(t)+f_{p r}(t)\right), \\
c_{q r}(t)=10\left(\gamma f_{q r}(t)+f_{o q}(t)\right), \\
c_{o q}(t)=\left(\gamma f_{o q}(t)+f_{q r}(t)\right)+50, \\
c_{p r}(t)=\left(\gamma f_{p r}(t)+f_{o p}(t)\right)+50,
\end{gathered}
$$

where $c_{i j}(t)$ is the travel cost on link $i j$ at time $t, f_{i j}(t)$ is the flow on link $i j$ at time $t, \gamma$ is the scaling parameter which 


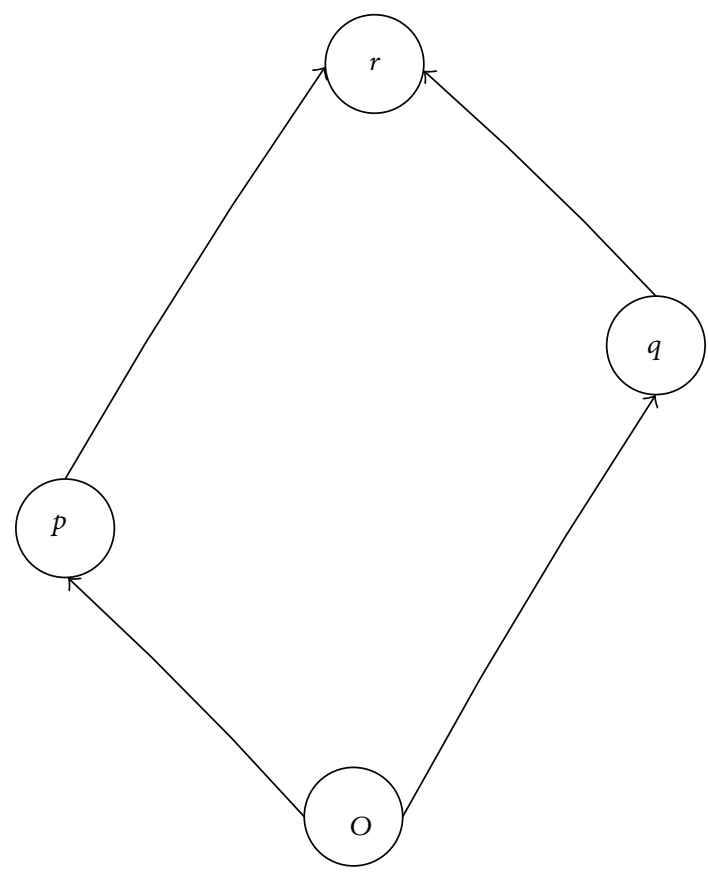

FIGURE 1: The four-link network.

differentiates the influence between the given link only and others, generally, $\gamma \geq 1$. In the four-link network, there are two paths from the origin $o$ to the destination $r$ and the costs along the paths are given as follows:

$$
\begin{aligned}
& C_{1}(t)=c_{o p}(t)+c_{p r}(t)=11(\gamma+1) f_{1}(t)+50, \\
& C_{2}(t)=c_{o q}(t)+c_{q r}(t)=11(\gamma+1) f_{2}(t)+50,
\end{aligned}
$$

where $C_{k}(t)$ is the travel cost from $o$ to $r$ along path $k$ at time $t$; in addition,

$$
\begin{aligned}
& f_{1}(t)=f_{o p}(t)=f_{p r}(t), \\
& f_{2}(t)=f_{o q}(t)=f_{q r}(t),
\end{aligned}
$$

where $f_{k}(t)$ is the flow from $o$ to $r$ along path $k$ at time $t$, the total demand satisfies the following conservation of the flow: $d_{w}(t)=f_{1}(t)+f_{2}(t)$. According to the definition of the UE, it is easy to get

$$
\begin{gathered}
f_{1}^{*}(t)=f_{2}^{*}(t)=\frac{t}{2}, \\
C_{1}(t)=C_{2}(t)=\frac{11(\gamma+1) t}{2}+50,
\end{gathered}
$$

where $f_{k}^{*}(t)$ is the equilibrium flow from $o$ to $r$ along path $k$ at time $t$. Hence, the total system travel time under DUE before

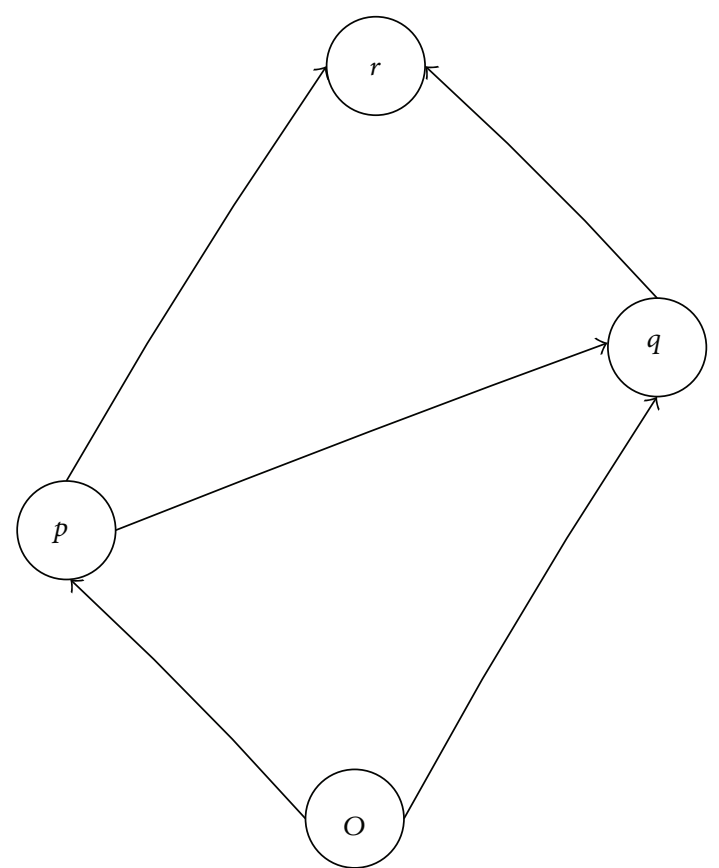

FIgURE 2: The five-link network.

the addition of the new link is given as follows:

$$
C^{4}(t)=\frac{11(\gamma+1) t^{2}}{2}+50 t
$$

where $C^{4}(t)$ is the total system travel cost under DUE for the four-link network.

\subsubsection{The Equilibrium Solution of the Five-Link Network under} DUE. After adding the link $p q$, there appears a new path opqr from $o$ to $r$ in Figure 2.

The link cost functions are given as follows:

$$
\begin{gathered}
c_{o p}(t)=10\left(\gamma f_{o p}(t)+f_{p r}(t)+f_{p q}(t)\right), \\
c_{q r}(t)=10\left(\gamma f_{q r}(t)+f_{o q}(t)+f_{p q}(t)\right), \\
c_{o q}(t)=\left(\gamma f_{o q}(t)+f_{q r}(t)\right)+50, \\
c_{p r}(t)=\left(\gamma f_{p r}(t)+f_{o p}(t)\right)+50, \\
c_{p q}(t)=\left(\gamma f_{p q}(t)+f_{o p}(t)+f_{q r}(t)\right)+10 .
\end{gathered}
$$

The traffic flow on each link is as follows:

$$
\begin{gathered}
f_{o p}(t)=f_{1}(t)+f_{3}(t), \\
f_{p r}(t)=f_{1}(t), \\
f_{o q}(t)=f_{2}(t), \\
f_{q r}(t)=f_{2}(t)+f_{3}(t), \\
f_{p q}(t)=f_{3}(t) .
\end{gathered}
$$


The costs on the paths are

$$
\begin{gathered}
C_{1}(t)=11(\gamma+1) f_{1}(t)+(10 \gamma+11) f_{3}(t)+50, \\
C_{2}(t)=11(\gamma+1) f_{2}(t)+(10 \gamma+11) f_{3}(t)+50, \\
C_{3}(t)=(10 \gamma+1)\left(f_{1}(t)+f_{2}(t)\right)+(21 \gamma+22) f_{3}(t)+10 .
\end{gathered}
$$

The total demand satisfies the conservation of the flow as follows: $d_{w}(t)=f_{1}(t)+f_{2}(t)+f_{3}(t)$.

The variational inequality of the five-link dynamic traffic network over $t \in[0, T]$ is given as follows:

$$
\begin{aligned}
\int_{0}^{T}( & \left.11(\gamma+1) f_{1}^{*}(t)+(10 \gamma+11) f_{3}^{*}(t)+50\right) \\
\times & \left(f_{1}(t)-f_{1}^{*}(t)\right) \\
+ & \left(11(\gamma+1) f_{2}^{*}(t)+(10 \gamma+11) f_{3}^{*}(t)+50\right) \\
\quad \times & \left(f_{2}(t)-f_{2}^{*}(t)\right) \\
+ & \left((10 \gamma+1)\left(f_{1}^{*}(t)+f_{2}^{*}(t)\right)\right. \\
& \left.\quad+(21 \gamma+22) f_{3}^{*}(t)+10\right)\left(f_{3}(t)-f_{3}^{*}(t)\right) d t \geq 0 .
\end{aligned}
$$

Because

$$
\begin{aligned}
d_{w}(t)= & f_{1}(t)+f_{2}(t)+f_{3}(t), \\
d_{w}(t)= & f_{1}^{*}(t)+f_{2}^{*}(t)+f_{3}^{*}(t), \\
& f_{1}^{*}(t)=f_{2}^{*}(t),
\end{aligned}
$$

we have

$$
\begin{gathered}
\int_{0}^{T}\left((13 \gamma+11) f_{1}^{*}(t)-11(\gamma+1) t+40\right) \\
\quad \times\left(f_{1}(t)+f_{2}(t)-2 f_{1}^{*}(t)\right) d t \geq 0
\end{gathered}
$$

if $f_{1}^{*}(t)=0$, and we have $-11(\gamma+1) t+40 \geq 0$, then $t \leq$ $40 / 11(\gamma+1)$; if $f_{3}^{*}(t)=0$, and at this time, $f_{1}^{*}(t)=t / 2$, then $t>80 /(9 \gamma+11)$; when $t \in(40 / 11(\gamma+1), 80 /(9 \gamma+11)]$,

$$
\begin{gathered}
f_{1}^{*}(t)=f_{2}^{*}(t)=\frac{11(\gamma+1) t-40}{13 \gamma+11}, \\
f_{3}^{*}(t)=\frac{-(9 \gamma+11) t+80}{13 \gamma+11} .
\end{gathered}
$$

Assume $\gamma=2$; the equilibrium flow of the five-link network is pictured in Figure 3, in ranges I and II [0,33/40], only the new path is used; in range III $(33 / 40,80 / 29)$, all three are used; in range IV $[80 / 29,+\infty)$, only the first two paths are used; that is, the third path is never used when $t>80 / 29$.

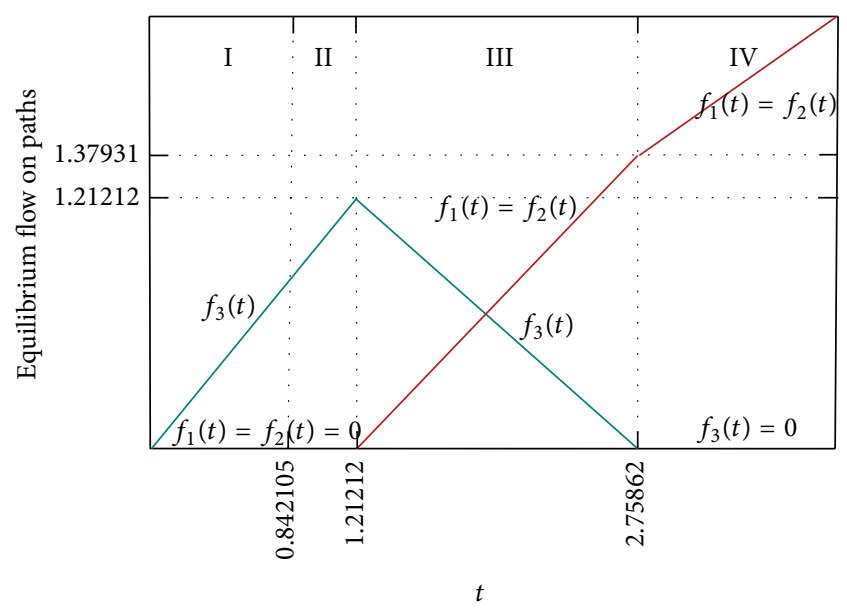

FIGURE 3: The equilibrium flow of the dynamic network.

Corresponding to different ranges, the total travel cost of five-link network is given as follows:

$C^{5}(t)$

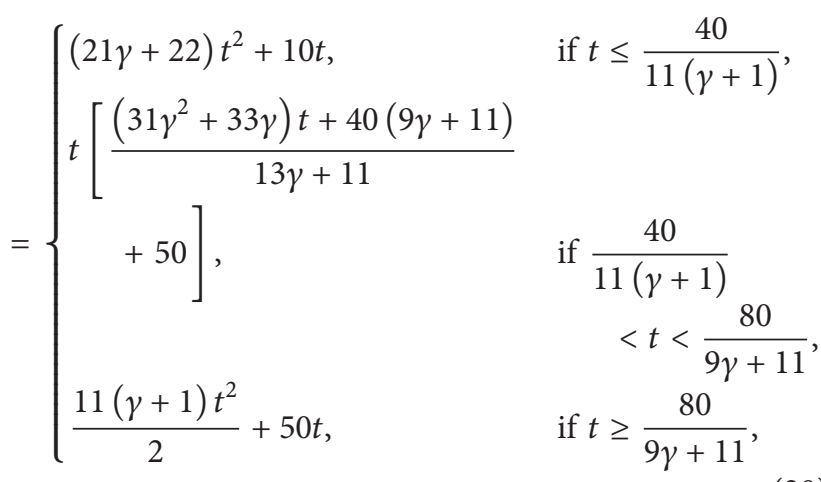

where $C^{5}(t)$ is the total system travel cost under DUE for the five-link network.

3.1.3. The Paradox of Dynamic Traffic Networks. Letting $C^{5}(t)>$ $C^{4}(t)$, we get $t \in(80 /(31 \gamma+33), 80 /(9 \gamma+11))$; letting $\gamma=2$, the paradox occurs in ranges II and III in Figure 3.

In order to capture the trend of the paradox when we take different values of $\gamma$, we give the definition of the average difference of two functions $F_{1}, F_{2}$ in range $[a, b]((a, b][a, b)$, $(a, b)), a>b$ as follows:

$$
D\left(F_{1}, F_{2}\right)=\frac{\left|F_{1}-F_{2}\right|}{b-a} .
$$

We assume that the severity of the paradox is proposal to the average difference and use of the average difference of $C^{4}, C^{5}$ in range $(80 /(31 \gamma+33), 80 /(9 \gamma+11))$ to represent the severity of the paradox.

In Figure 4, we discuss the situations when $\gamma=1,2,5$, respectively, and find that the areas where the paradox occurs are different when the values of $\gamma$ are different; thus we may take some measures to control the influence between the links 


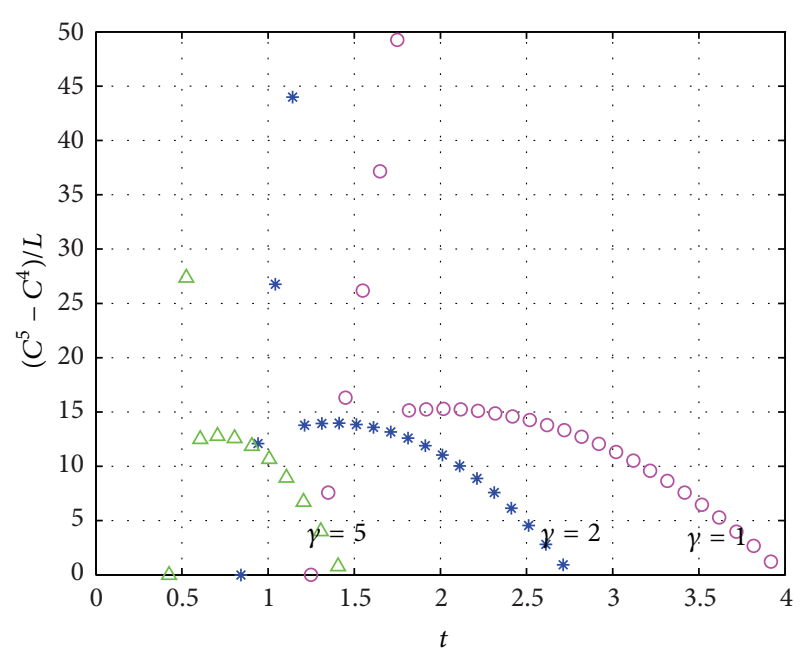

FIGURE 4: The severity that the paradox occurs when $\gamma$ is different.

as time and traffic demand change in order to avoid or decrease the occurrence of the paradox; in addition, in most range, the greater $\gamma$ is, the smaller the range of the traffic demand in which the paradox occurs is; that is, the severity and the range in which the paradox occurs increase with the influence of other links decreasing, which reminds us to let down the influence between links as much as possible if we wish to decrease the occurrence of the paradox.

3.1.4. The Effect of the Adding Link under DSO. As known, when the given link cost is a function of the flow on the given link only, adding a new link did not reduce the total system travel time even under SO [18]. When the link cost does not only depend on the flow on the given link only, we discuss whether the phenomenon occurs or not in the following. As we know, DSO is obtained by charging users the marginal cost of traveling (see formula (6)); for the link cost in this work, the corresponding path marginal cost equations are given as follows:

$$
\begin{gathered}
c_{o p}^{\prime}(t)=10\left(2 \gamma f_{o p}(t)+f_{p r}(t)+f_{p q}(t)\right), \\
c_{q r}^{\prime}(t)=10\left(2 \gamma f_{q r}(t)+f_{o q}(t)+f_{p q}(t)\right), \\
c_{o q}^{\prime}(t)=\left(2 \gamma f_{o q}(t)+f_{q r}(t)\right)+50, \\
c_{p r}^{\prime}(t)=\left(2 \gamma f_{p r}(t)+f_{o p}(t)\right)+50, \\
c_{p q}^{\prime}(t)=\left(2 \gamma f_{p q}(t)+f_{o p}(t)+f_{q r}(t)\right)+10 .
\end{gathered}
$$

The cost on the paths is as follows:

$$
\begin{gathered}
C_{1}^{\prime}(t)=11(2 \gamma+1) f_{1}(t)+(20 \gamma+11) f_{3}(t)+50, \\
C_{2}^{\prime}(t)=11(2 \gamma+1) f_{2}(t)+(20 \gamma+11) f_{3}(t)+50, \\
C_{3}^{1}(t)=(20 \gamma+11)\left(f_{1}(t)+f_{2}(t)\right) \\
+2(21 \gamma+11) f_{3}(t)+10 .
\end{gathered}
$$

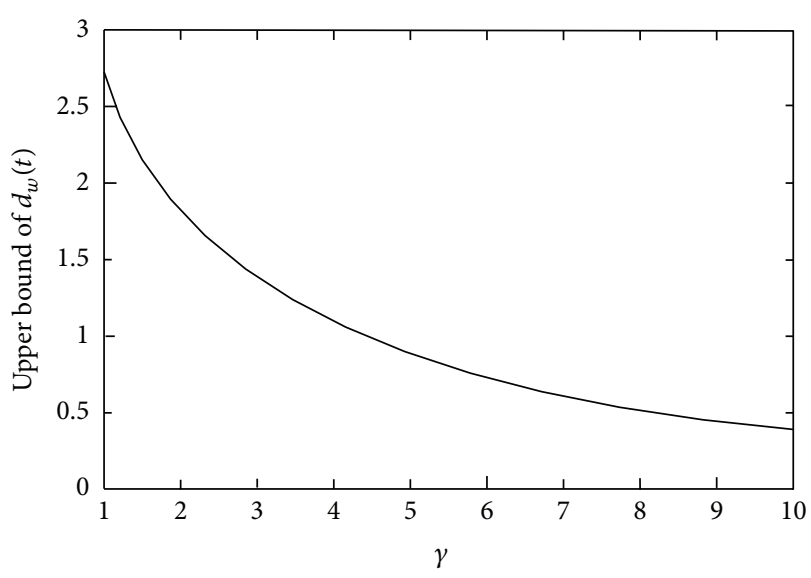

FIgURE 5: The bound above which an adding link cannot make the total costs increase under DSO.

Then the variational inequality of the five-link dynamic traffic network over $t \in[0, T]$ under DSO is given as follows:

$$
\begin{aligned}
\int_{0}^{T} & \left(11(2 \gamma+1) f_{1}^{*}(t)+(20 \gamma+11) f_{3}^{*}(t)+50\right) \\
& \times\left(f_{1}(t)-f_{1}^{*}(t)\right) \\
& +\left(11(2 \gamma+1) f_{2}^{*}(t)+(20 \gamma+11) f_{3}^{*}(t)+50\right) \\
& \times\left(f_{2}(t)-f_{2}^{*}(t)\right) \\
+ & \left((20 \gamma+11)\left(f_{1}^{*}(t)+f_{2}^{*}(t)\right)\right. \\
& \left.\quad+2(21 \gamma+11) f_{3}^{*}(t)+10\right) \\
& \times\left(f_{3}(t)-f_{3}^{*}(t)\right) d t \geq 0 .
\end{aligned}
$$

Because of formula (17), the equation can be given as follows:

$$
\begin{gathered}
\int_{0}^{T}\left((26 \gamma+11) f_{1}^{*}(t)-11(2 \gamma+1) t+40\right) \\
\quad \times\left(f_{1}(t)+f_{2}(t)-2 f_{1}^{*}(t)\right) d t \geq 0 .
\end{gathered}
$$

Let $f_{3}^{*}(t)=0$; at this time, $f_{1}^{*}(t)=f_{2}^{*}(t)=t / 2$ and $(26 \gamma+$ $11)(t / 2)-11(2 \gamma+1) t+40 \geq 0$; we have $t \leq 80 /(18 \gamma+11)$; when $t \in[0,80 /(18 \gamma+11)]$, the adding link makes sense under DSO.

In the following, we give the trend of the upper bound under which the adding link works under DSO as the parameter $\gamma$ changes in Figure 5 and find that the bound becomes smaller as $\gamma$ increases, which explains that the less the influence of the other links is, the less the possibility that the adding link works under DSO, which warns us of improving the influence between the links appropriately if we want to make the adding link work under DSO.

3.1.5. Relationship between the Total Cost under DUE and DSO. There is much research about the relationship of the total costs under different assignments, in which [22] we know that the solution under DUE and that under DSO are 


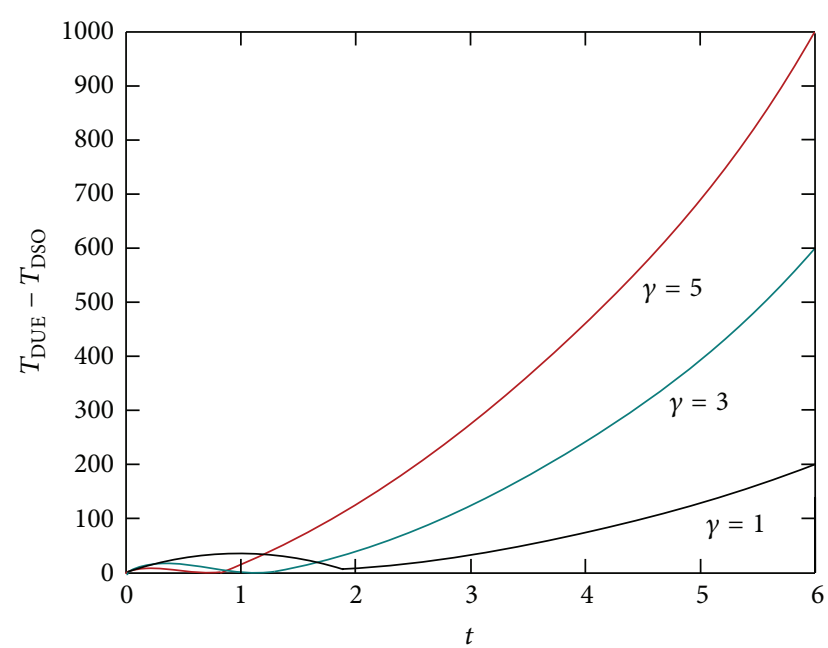

FIGURE 6: Trend of $T_{\mathrm{DUE}}-T_{\mathrm{DSO}}$ with $t$ changing when the values of $\gamma$ are different.

close under the free flow state, and the difference between both assignments is great when the traffic is congested in the kind of networks. In this section, we discuss the situation of the dynamic network when the link cost is related to the flow of the link and the flow of other links. Define $T_{\mathrm{DUE}}-T_{\mathrm{DSO}}$ as the distance of the total costs between DUE and DSO. In this paper, we capture the gap of the total costs between the two assignments using the distance of the total costs between DUE and DSO. In Figure 6, we give the trend of the gap when $\gamma$ is 1,3 , and 5 , respectively and find that the gap increases with the time being over and demand accumulating; in addition, the gap increases with $\gamma$ increasing in the area of the great demand. It shows, with $\gamma$ increasing, that the influence of other links decreases; that is, it is reverse with the idea of DSO ignoring the influence of other links; thus the gap increases.

3.2. Robustness of the Dynamic Network. The network robustness has been widely investigated in many literatures, in which the importance identification of the network components is an important branch $[23,24]$. In this section, we use the relative total cost index to determine the importance of the components of the dynamic network.

The total cost on the link $a$ is denoted as follows:

$$
\widehat{c}_{i j}(t)=c_{i j}(t) f_{i j}(t),
$$

where $\widehat{c}_{i j}(t)$ is the total cost on the link $i j$ at time $t, c_{i j}(t)$ is the unit cost on the link $i j$ at the time $t$, and $f_{i j}(t)$ is the flow on the link $i j$ at time $t$. Hence, the total cost TC $(t)$ on a network at time $t$ is given by

$$
\mathrm{TC}(t)=\sum_{i j \in L} \widehat{c}_{o j}(t)
$$

where $L$ is the set of the links. The link flows $f(t)$ satisfy the nonnegativity and conservation of the flow conditions. Then we have the relative total cost index as follows:

$$
\Gamma^{l}(t)=\frac{\mathrm{TC}_{\mathrm{G}-l}(t)-\mathrm{TC}(t)}{\mathrm{TC}(t)}
$$

where $\Gamma^{l}(t)$ denotes the important indicator for the link $l$ under the DUE at time $t$ and $\mathrm{TC}_{G-l}(t)$ denotes the total cost generated under DUE when the link $l$ is removed from the network at time $t$. Similarly, the importance indicator for the node $M$ is denoted as follows:

$$
\Gamma^{M}(t)=\frac{\mathrm{TC}_{\mathrm{G}-M}(t)-\mathrm{TC}(t)}{\mathrm{TC}(t)},
$$

where $\Gamma^{M}(t)$ is the importance indicator for the node $M$ under DUE at time $t, \mathrm{TC}_{G-M}(t)$ is the total cost when the node $M$ is removed from the network under DUE at time $t$.

In the following, we use the network in Figure 2 and assume $\gamma=2$ to investigate the importance of components of the dynamic network. During the process of the calculation, it can be found that the different traffic demands correspond to different traffic assignments with different importance components; the range of $t$ is divided into four different ranges as follows.

Demand Range I:

$$
t \in\left[0, \frac{16}{19}\right)
$$

We know that, in this demand range, only the third path is used at the equilibrium; hence, we have the equilibrium path flow pattern $f(t)_{1}=f_{2}(t)=0, f_{3}(t)=t$, and the total cost is $\mathrm{TC}(t)=64 t^{2}+10 t$.

Demand Range II:

$$
t \in\left[\frac{16}{19}, \frac{40}{33}\right) .
$$

We know that, in this demand range, only the third path is used at the equilibrium, hence; we have the equilibrium path flow pattern $f(t)_{1}=f_{2}(t)=0, f_{3}(t)=t$, and the total cost is $\mathrm{TC}(t)=64 t^{2}+10 t$.

Demand Range III:

$$
t \in\left[\frac{40}{33}, \frac{80}{29}\right) .
$$

We know that, in this range of the demand, all three paths are used at the equilibrium; we now have that $f_{1}(t)=f_{2}(t)=$ $(33 t-40) / 37, f_{3}(t)=(80-29 t) / 37$, and the total cost is $\mathrm{TC}(t)=\left(190 t^{2}+2640 t\right) / 37$.

Demand Range IV:

$$
t \in\left[\frac{80}{29},+\infty\right)
$$

We know that only the first two paths are used at the equilibrium and the Braess' paradox vanishes. Hence, we have the equilibrium path flow pattern $f_{1}(t)=f_{2}(t)=t / 2, f_{3}(t)=$ 0 , and the total cost is $\operatorname{TC}(t)=\left(33 t^{2} / 2\right)+50 t$.

The ranking of the component is the sequence of its important value. Then the important values and rankings of different components in different ranges are given in Tables 1 , 2,3 , and 4 .

From Tables 1-4, the important values and the rankings of the different components are different; at the same time, 
TABLE 1: Important values of links in different ranges.

\begin{tabular}{ccccc}
\hline & I & II & III & IV \\
\hline op & $\frac{40-31 t}{64 t+10}$ & $\frac{40-31 t}{64 t+10}$ & $\frac{1031 t-1160}{190 t+3010}$ & $\frac{33 t}{33 t+100}$ \\
$o q$ & 0 & 0 & $\frac{35937 t-43560}{6650 t+105350}$ & $\frac{1147 t-160}{1155 t+3500}$ \\
pr & 0 & 0 & $\frac{35937 t-43560}{6650 t+105350}$ & $\frac{1147 t-160}{1155 t+3500}$ \\
qr & $\frac{40-31 t}{64 t+10}$ & $\frac{40-31 t}{64 t+10}$ & $\frac{1031 t-1160}{190 t+3010}$ & $\frac{33 t}{33 t+100}$ \\
$p q$ & $\frac{5(18-19 t)}{4(32 t+5)}$ & $\frac{5(18-19 t)}{4(32 t+5)}$ & $\frac{841 t-2320}{380 t+6020}$ & 0 \\
\hline
\end{tabular}

TABLE 2: Important rankings of links in different ranges.

\begin{tabular}{lllcc}
\hline & I & II & III & IV \\
\hline$o p$ & 1 & 1 & 1 & 1 \\
$o q$ & 3 & 2 & 2 & 2 \\
$p r$ & 3 & 2 & 2 & 2 \\
$q r$ & 1 & 1 & 1 & 1 \\
$p q$ & 2 & 3 & 3 & 3 \\
\hline
\end{tabular}

TABLE 3: Important values of nodes in different ranges.

\begin{tabular}{ccccc}
\hline & I & II & III & IV \\
\hline$o$ & $+\infty$ & $+\infty$ & $+\infty$ & $+\infty$ \\
$p$ & $\frac{40-31 t}{64 t+10}$ & $\frac{40-31 t}{64 t+10}$ & $\frac{1031 t-1160}{190 t+3010}$ & $\frac{33 t}{33 t+100}$ \\
$q$ & $\frac{40-31 t}{64 t+10}$ & $\frac{40-31 t}{64 t+10}$ & $\frac{1031 t-1160}{190 t+3010}$ & $\frac{33 t}{33 t+100}$ \\
$r$ & $+\infty$ & $+\infty$ & $+\infty$ & $+\infty$ \\
\hline
\end{tabular}

TABLE 4: Important rankings of nodes in different ranges.

\begin{tabular}{ccccc}
\hline & I & II & III & IV \\
\hline$o$ & 1 & 1 & 1 & 1 \\
$p$ & 2 & 2 & 2 & 2 \\
$q$ & 2 & 2 & 2 & 2 \\
$r$ & 1 & 1 & 1 & 1 \\
\hline
\end{tabular}

the important rankings of the component are different in different ranges of the time, which show the robustness of the network changes when different components collapse.

Owing to considering the influence of other links and using different efficiency measures, we find that the important values of the corresponding nodes and links are different from the results of Nagurney and Qiang [25], but the important ranking of the corresponding components is the same as their results; there are two possible reasons as follows: (i) the purpose and essence of the different efficiency measures are consistent; (ii) in this work, we assume $\gamma=2$; that is, the influence of other links is secondary.

In addition, from Sections 3.1.3 and 3.2, we find that the importance of the adding link is small in the area that Braess' paradox occurs and the adding link is not used, which shows that the robustness of the network is related to whether the paradox occurs. Based on the preceding study, we consider the influence of other links in this paper, which better provide the guidance of the traffic assignment.

\section{Conclusion}

In this work, we investigate the paradox phenomenon and the robustness of the dynamic traffic network, in which the cost influenced by other link flows is considered. The four-link network and the five-link network are used in the analysis, which reveal that the paradox occurs only in certain range. The difference of the total costs between DUE and DSO changes as the traffic demand changes. The important values and important rankings of network components are changing at different demand levels. The results show that whether the paradox occurs or not and the robustness are both affected by the traffic demand, and the robustness of the network is related to the occurrence of the paradox, which provides a theoretical basis to the traffic assignment. The more complex networks are closer to the reality and needed to be further investigated.

\section{Acknowledgments}

The authors would like to thank the anonymous referees for their useful comments on this paper. This work is supported by the National Natural Science Foundation of China (71171124, 10871219).

\section{References}

[1] J. R. Birge and J. K. Ho, "Optimal flows in stochastic dynamic networks with congestion," Operations Research, vol. 41, no. 1, pp. 203-216, 1993.

[2] B. N. Janson, "Dynamic traffic assignment for urban road networks," Transportation Research B, vol. 25, no. 2-3, pp. 143$161,1991$.

[3] B.-W. Wie, T. L. Friesz, and R. L. Tobin, "Dynamic user optimal traffic assignment on congested multidestination networks," Transportation Research B, vol. 24, no. 6, pp. 431-442, 1990.

[4] B. Ran, D. E. Boyce, and L. J. LeBlanc, "New class of instantaneous dynamic user-optimal traffic assignment models," Operations Research, vol. 41, no. 1, pp. 192-202, 1993.

[5] H.-K. Chen and C.-F. Hsueh, "A model and an algorithm for the dynamic user-optimal route choice problem," Transportation Research B, vol. 32, no. 3, pp. 219-234, 1998.

[6] R. Jayakrishnan, W. K. Tsai, and A. Chen, "A dynamic traffic assignment model with traffic-flow relationships," Transportation Research C, vol. 3, no. 1, pp. 51-72, 1995.

[7] X. J. Nie, The study of dynamic user-equilibrium traffic assignment [Ph.D. thesis], University of California, Davis, Calif, USA, 2003.

[8] S. C. Dafermos and F. T. Sparrow, "The traffic assignment problem for a general network," Journal of Research of the National Bureau of Standards, vol. 73, pp. 91-118, 1969.

[9] M. J. Smith, “The existence, uniqueness and stability of traffic equilibria," Transportation Research B, vol. 13, no. 4, pp. 295304, 1979. 
[10] M. Ng and S. T. Waller, "A dynamic route choice model considering uncertain capacities," Computer-Aided Civil and Infrastructure Engineering, vol. 27, no. 4, pp. 231-243, 2012.

[11] Q. Meng and H. L. Khoo, "A computational model for the probit-based dynamic stochastic user optimal traffic assignment problem," Journal of Advanced Transportation, vol. 46, no. 1, pp. 80-94, 2012.

[12] S. Dafermos, "Traffic equilibrium and variational inequalities," Transportation Science, vol. 14, no. 1, pp. 42-54, 1980.

[13] D. Braess, "Über ein paradoxon aus der verkehrsplanung," Unternehmensforschung, vol. 12, pp. 258-268, 1968.

[14] S. Dafermos and A. Nagurney, "On some traffic equilibrium theory paradoxes," Transportation Research B, vol. 18, no. 2, pp. 101-110, 1984.

[15] T. Akamatsu and B. Heydecker, "Detecting dynamic traffic assignment capacity paradoxes in saturated networks," Transportation Science, vol. 37, no. 2, pp. 123-138, 2003.

[16] T. Roughgarden, Selfish Routing and the Price of Anarchy, MIT Press, 2005.

[17] X. Zhang and H. M. Zhang, "Simultaneous departure time/ route choices in queueing networks and a novel paradox," Networks and Spatial Economics, vol. 10, no. 1, pp. 93-112, 2010.

[18] E. I. Pas and S. L. Principio, "Braess' paradox: some new insights," Transportation Research B, vol. 31, no. 3, pp. 265-276, 1997.

[19] R. Arnott, A. de Palma, and R. Lindsey, "Properties of dynamic traffic equilibrium involving bottlenecks, including a paradox and metering," Transportation Science, vol. 27, no. 2, pp. 148$160,1993$.

[20] A. Hallefjord, K. Jörnsten, and S. Storøy, "Traffic equilibrium paradoxes when travel demand is elastic," Asia-Pacific Journal of Operational Research, vol. 11, no. 1, pp. 41-50, 1994.

[21] X. Zhang, W. H. K. Lam, and H.-J. Huang, "Braess's paradoxes in dynamic traffic assignment with simultaneous departure time and route choices," Transportmetrica, vol. 4, no. 3, pp. 209-225, 2008.

[22] J. N. Prashker and S. Bekhor, "Some observations on stochastic user equilibrium and system optimum of traffic assignment," Transportation Research B, vol. 34, no. 4, pp. 277-291, 2000.

[23] A. Nagurney and Q. Qiang, "A relative total cost index for the evaluation of transportation network robustness in the presence of degradable links and alternative travel behavior," International Transactions in Operational Research, vol. 16, no. 1, pp. 49-67, 2009.

[24] A. Nagurney, Q. Qiang, and L. S. Nagurney, "Environmental impact assessment of transportation networks with degradable links in an era of climate change," International Journal of Sustainable Transportation, vol. 4, no. 3, pp. 154-171, 2010.

[25] A. Nagurney and Q. Qiang, "A network efficiency measure for congested networks," Europhysics Letters, vol. 79, no. 3, Article ID 38005, 5 pages, 2007. 


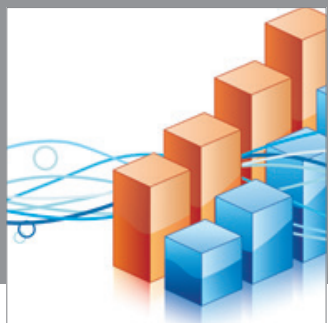

Advances in

Operations Research

mansans

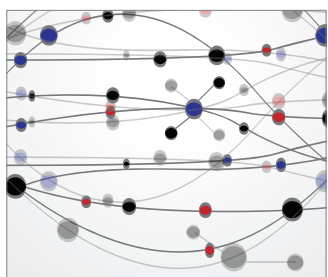

The Scientific World Journal
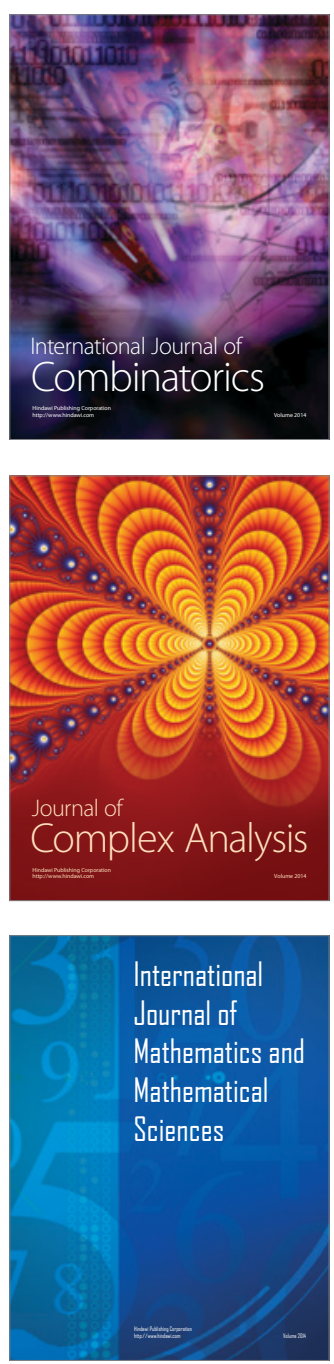
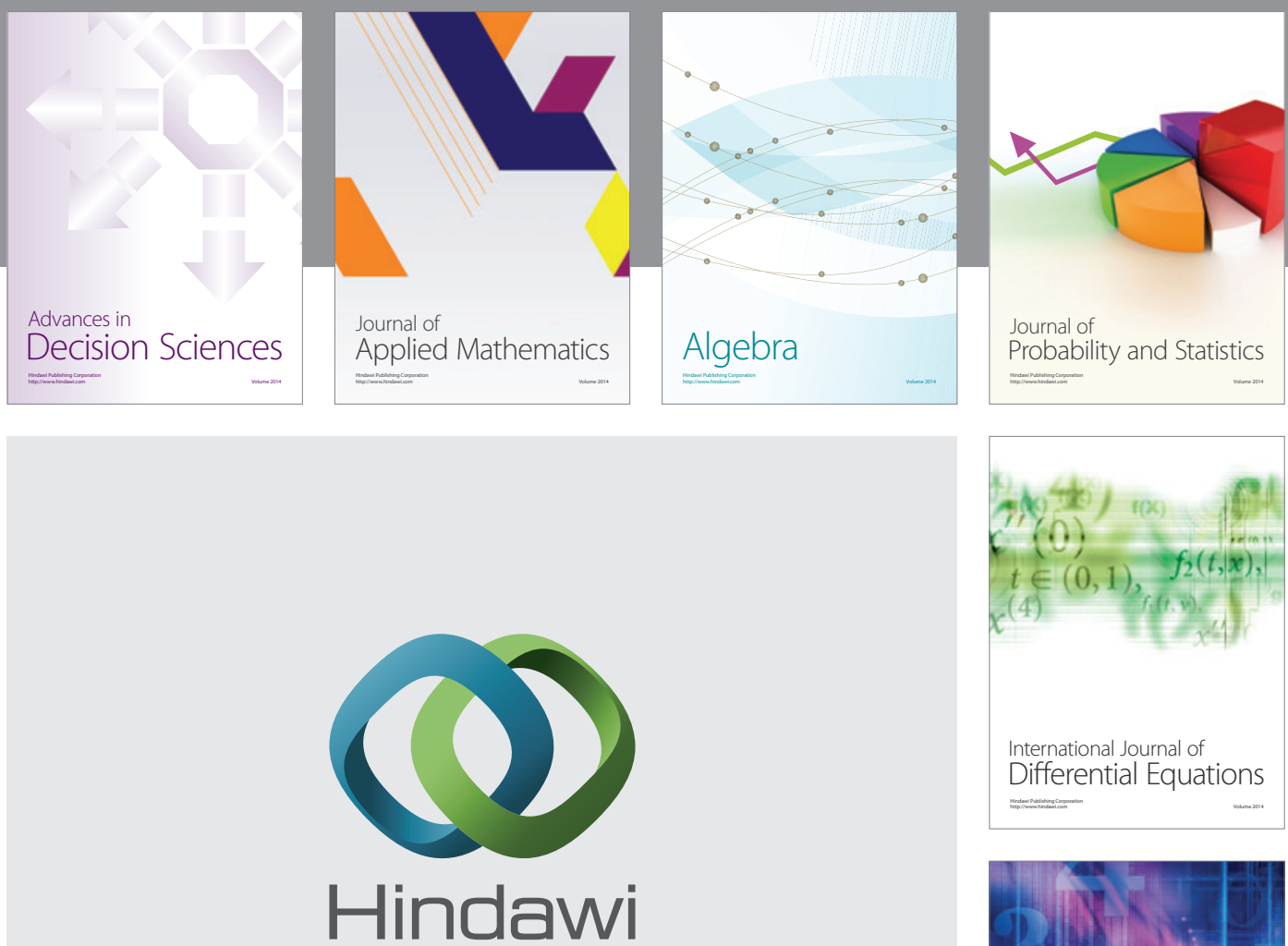

Submit your manuscripts at http://www.hindawi.com
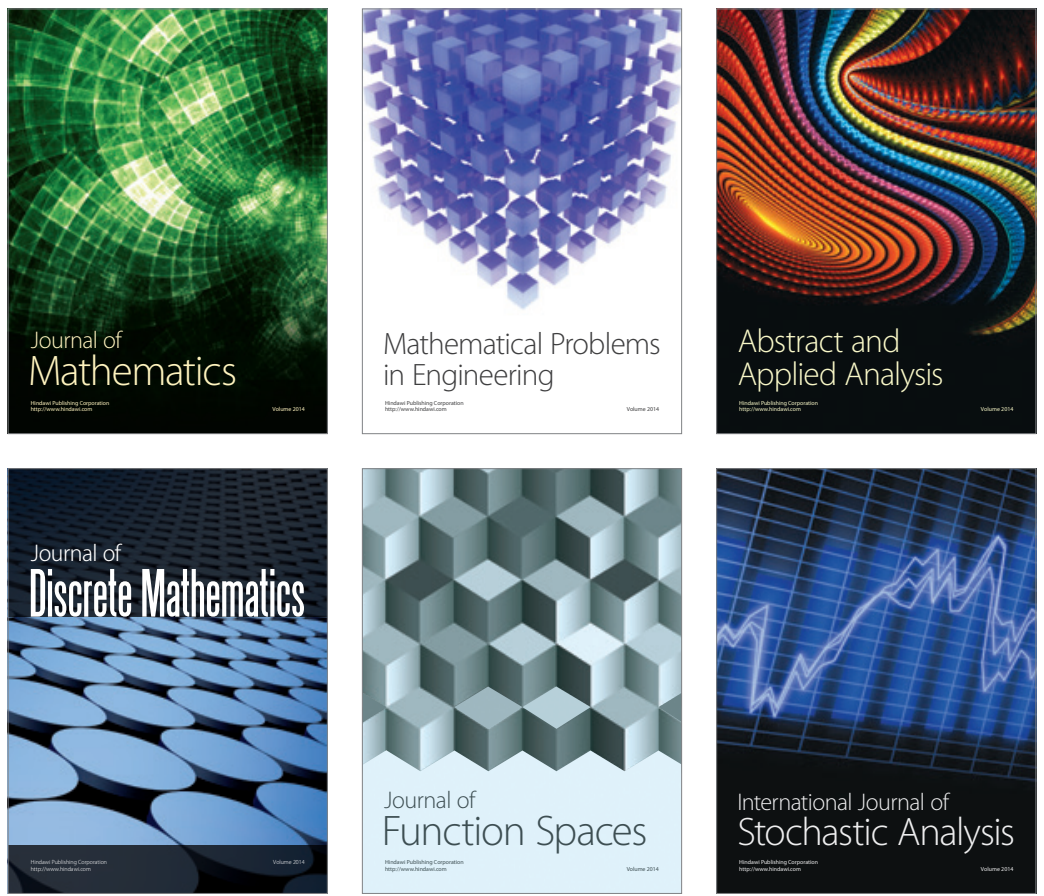

Journal of

Function Spaces

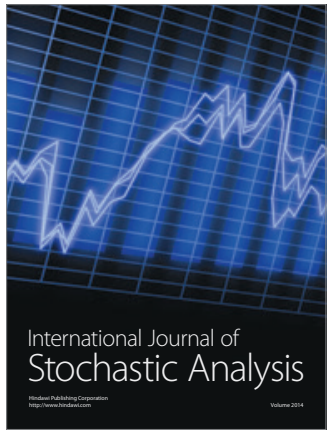

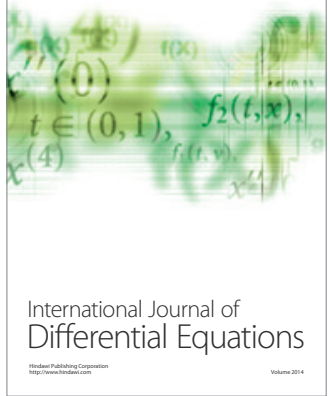
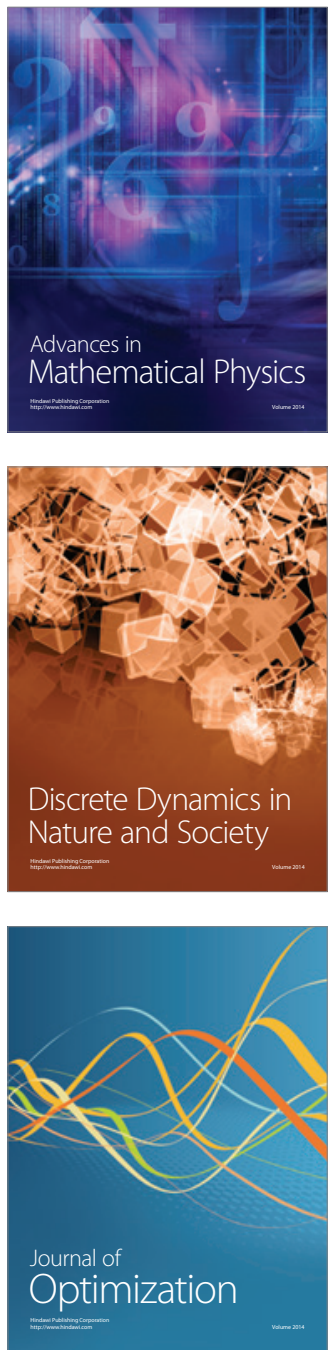